\title{
Preface to special issue: EXPRESS/SOS $2016+2017$
}

\author{
Kirstin Peters ${ }^{1} \cdot$ Simone Tini ${ }^{2}$ \\ Published online: 19 September 2019 \\ ๑) Springer-Verlag GmbH Germany, part of Springer Nature 2019
}

This issue of Acta Informatica contains a selection of papers presented at

- The Combined 23rd International Workshop on Expressiveness in Concurrency and 13th Workshop on Structural Operational Semantics (EXPRESS/SOS 2016), a satellite event of CONCUR'16, held on August 22nd, 2016 in Québec City, Canada, and

- The Combined 24th International Workshop on Expressiveness in Concurrency and 14th Workshop on Structural Operational Semantics (EXPRESS/SOS 2017), a satellite event of CONCUR'17, held on September 4th, 2017 in Berlin, Germany.

The EXPRESS workshops aim at bringing together researchers interested in the expressiveness of various formal systems and semantic notions, particularly in the field of concurrency. Their focus has traditionally been on the comparison between programming concepts (such as concurrent, functional, imperative, logic and object-oriented programming) and between mathematical models of computation (such as process algebras, Petri nets, event structures, modal logics, and rewrite systems) on the basis of their relative expressive power. The EXPRESS workshop series has run successfully since 1994 and over the years this focus has become broadly construed.

The SOS workshops aim at being a forum for researchers, students and practitioners interested in new developments, and directions for future investigation, in the field of structural operational semantics. One of the specific goals of the SOS workshop series is to establish synergies between the concurrency and programming language communities working on the theory and practice of SOS. Reports on applications of SOS to other fields are also most welcome, including: modelling and analysis of biological systems, security of computer systems programming, modelling and analysis of embedded systems, specification of middle-ware and coordination languages, programming language semantics and implementation, static analysis software and hardware verification, semantics for domain-specific languages and model-based engineering.

This issue contains four extended and revised versions of selected papers presented at EXPRESS/SOS' 16 and EXPRESS/SOS'17. All papers have undergone a reviewing process in accordance with the standards of Acta Informatica. We would like to thank the authors for

\footnotetext{
Kirstin Peters

kirstin.peters@tps.tu-darmstadt.de

$凶$ Simone Tini

simone.tini@uninsubria.it

1 TU Darmstadt, Darmstadt, Germany

2 University of Insubria, Como, Italy
} 
their efforts in producing the extended versions contained in this journal issue. We are very grateful to the expert reviewers for their very careful reading; their help has been crucial in producing this issue. We would also like to thank the program committee of EXPRESS/SOS' 16 and EXPRESS/SOS'17, for the evaluation of the proceeding versions of these papers. Last but not the least, our special thanks go to the Editor-in-Chief for the opportunity of publishing in Acta Informatica.

Kirstin Peters

Simone Tini

Darmstadt and Como, 2019

Publisher's Note Springer Nature remains neutral with regard to jurisdictional claims in published maps and institutional affiliations. 